\title{
Analyticity Properties of the Surface Free Energy of the Ising Model
}

\author{
C.-E. Pfister ${ }^{1}$ and O. Penrose ${ }^{2}$ \\ ${ }^{1}$ Département de Mathématiques, EPFL-Lausanne, CH-1015 Lausanne, Switzerland \\ ${ }^{2}$ Department of Mathematics, Heriot-Watt University, Riccarton, Edinburgh, EH144AS, UK
}

\begin{abstract}
For an Ising ferromagnet with nearest-neighbour interactions of strength $K$ and surface magnetic field $h$, the surface free energy in the presence of a positively (or negatively) magnetized zero-field bulk phase is shown to be analytic in $h$ for $|\operatorname{Re} h|<K-\alpha / \beta$, where $\alpha=2.96 \ldots$ and $\beta$ is the inverse temperature. This puts the lower bound $K-\alpha / \beta$ on the values of $h$ at which wetting and layering transitions can take place.
\end{abstract}

\section{Introduction}

Surface phase transitions such as the wetting transition can be modelled by an Ising ferromagnet in which the magnetic field applied on a part of the boundary of the lattice is different from that in the bulk. (This is equivalent to a binary mixture on a lattice in contact with a wall which preferentially adsorbs one or the other of the two components, depending on the sign of the surface magnetic field $h$.) The surface and bulk magnetic fields, which may be in opposite directions, compete to determine the state near the surface. At temperatures $T$ below the bulk critical temperature $T_{c}$ this competition persists even in the limit of zero bulk field: if the part of the lattice far from the surface is in the negatively magnetized phase, corresponding to an infinitesimally small negative bulk field, then it is still necessary for the surface field $h$ to exceed a certain positive value $h_{w}(T)$ if the surface layer of positively magnetized phase near the surface is to invade the bulk. This "wetting" transition at $h=h_{w}$ was rigorously established by Abraham [1] for the two-dimensional case, and by Fröhlich and Pfister [2] for three or more dimensions. A summary of the known rigorous results for such models is given in [3].

One way to study possible phase transitions in the surface is to look at the surface free energy, whose precise definition is given later in this paper. It is important to realize that the surface free energy is a single-valued function of the parameters of the model (surface magnetic field, bulk magnetic field and temperature) only if there is a unique bulk phase. Therefore, with the bulk field held 
at zero and the temperature held below the critical temperature $T_{c}$, the surface free energy is two-valued: for a given value $h$ of the surface magnetic field there is one surface free energy $F^{+}(h)$ in the presence of a positively magnetized bulk phase, and a different one, $F^{-}(h)$, in the presence of a negatively magnetized bulk phase. Twenty years ago, McCoy and $\mathrm{Wu}$ [4] computed another surface free energy - call it $F^{p}(h)$ - for the two-dimensional model with zero bulk field and periodic boundary conditions. Below the critical temperature, this surface free energy, which is a continuous function of $h$, has a discontinuous first derivative at $h=0$, corresponding to a discontinuity in the surface magnetization. McCoy and Wu also found that the function $F^{p}(h)$ for $h>0$ could be extrapolated by analytic continuation through the singularity at $h=0$ to negative values of $h$. They interpreted the extrapolated function, call it $F^{p+}(h)$, in terms of hysteresis, i.e. as describing a possible metastable surface phase. Such a metastable phase would presumably be associated with some kind of first-order surface phase transition at $h=0 . \operatorname{In}[2,5]$, however, it was proved that the functions $F^{+}(h)$ and $F^{p+}(h)$ coincide for $h>0$ and it was conjectured that they also coincide for small negative values of $h$. In that case, the analyticity of $F^{p+}$ at $h=0$, found by McCoy and $\mathrm{Wu}$, has the following simple interpretation: provided we keep the bulk phase positively magnetized (e.g. through the imposition of "plus" boundary conditions at the parts of the boundary where $h$ does not act) the surface state varies analytically with $h$ near $h=0$. The discontinuity found by McCoy and Wu at $h=0$ then comes about not from a surface phase transition but from the one in the bulk: with periodic boundary conditions the bulk magnetization changes sign as $h$ passes through the value 0 , and so a discontinuous change in the properties of the surface is no surprise.

This interpretation, however, does not entirely dispose of the possibility of a metastable surface state at $h=0$; for in three or more dimensions the surface phase transitions are more complicated than in two, as shown by the existence of a firstorder layering transition [2], so that the analyticity found by McCoy and $\mathrm{Wu}$ for two dimensions might not persist in higher dimensions.

It is the purpose of this paper to give a general proof that $F^{+}$is analytic in $h$ at $h=0$ for sufficiently low temperatures. This shows that even for three or more dimensions there is no surface phase transition, and hence, presumably, no metastable surface state at $h=0$; it also confirms the conjecture of Fröhlich and Pfister that for small negative values of $h$ the functions $F^{+}(h)$ (defined by using "plus" boundary conditions) and $F^{p+}(h)$ (obtained by using periodic boundary conditions and analytic continuation from positive values of $h$ ) are identical. At the same time our method gives a new lower bound on $h_{w}(T)$ and (in three or more dimensions) on the value(s) of the surface field at which the layering transition occurs.

\section{The Model}

We consider a ferromagnetic Ising model defined on the semi-infinite $d$-dimensional lattice $\mathbb{L}=\mathbb{Z}^{d-1} \otimes \mathbb{Z}^{+}$. A point $i$ in $\mathbb{L}$ is labelled by two coordinates $(x, z)$ with $x$ in $\mathbb{Z}^{d-1}$ and $z$ in $\mathbb{Z}^{+}$. The first layer of the lattice is the layer $z=1$, and 
we denote it by $\Sigma$. For each $i$ we have a spin variable $s(i)$ in $\{1,-1\}$. The Hamiltonian is the formal sum

$$
H=-K \sum_{\langle i j\rangle} s(i) s(j)-h \sum_{i \in \Sigma} s(i),
$$

where the first summation is taken over pairs of nearest neighbour vertices of $\mathbb{L}$ and the second is taken over the vertices of $\Sigma$. The coupling constant $K$ is positive. Usually one introduces another coupling constant, say $J$, when $i$ and $j$ are both in the layer $z=1$, but for the sake of simplicity we have chosen $J=K$ here. However, all our results are valid without modification for $J>K$ and they can be extended to the case $0<J<K$.

Let us recall the definition of $F^{+}(\beta, h)$ given in [5]. Let $A(L, M)$ be the finite subset of IL defined by

$$
\Lambda(L, M)=\{(x, z):|x| \leqq L, 1 \leqq z \leqq M\},
$$

where $|x|=\max _{i}\left(\left|x_{i}\right|\right)$ with $x=\left(x_{1}, \ldots, x_{d-1}\right)$. Let $H_{A}^{+}$be the Hamiltonian of the finite system in $\Lambda(L, M)$ with "plus" boundary conditions. It is obtained by restricting the summations in (1.1) to terms where one or both of $i$ and $j$ is in $\Lambda(L, M)$, and putting $s(i)=1$ whenever $i$ is not in $\Lambda(L, M)$. The corresponding partition function at inverse temperature $\beta$ will be deroted by $Z_{L, M}^{+}(\beta, h)$. We also consider another copy of the model, in a box $\Lambda^{\prime}(L, M)$, which is obtained by reflecting $\Lambda(L, M)$ in the layer $z=\frac{1}{2}$. For both copies we choose "plus" boundary conditions, and for the box $A^{\prime}$ the magnetic field $h$ is applied in the layer $z=0$. We may put these two separate systems together to make a single composite system contained in the box

$$
\Omega(L, M)=\Lambda(L, M) \cup \Lambda^{\prime}(L, M)=\{(x, z):|x| \leqq L,-M+1 \leqq z \leqq M\} .
$$

The partition function of this composite system is $\left(Z_{L, M}^{+}\right)^{2}$. In the box $\Omega$ we also consider an Ising model with zero magnetic field, "plus" boundary conditions, and coupling constant $K$ for all bonds, including those joining $\Lambda$ to $\Lambda^{\prime}$. Let $Q_{L, M}^{+}$denote the corresponding partition function. Then the surface free energy for the finite box $\Lambda$ is defined as

$$
F_{L, M}^{+}(\beta, h)=\frac{-1}{2 \beta(2 L+1)^{d-1}} \ln \frac{\left(Z_{L, M}^{+}(\beta, h)\right)^{2}}{Q_{L, M}^{+}(\beta)},
$$

and the surface free energy for the infinite system is

$$
F^{+}(\beta, h)=\lim _{L, M \rightarrow \infty} F_{L, M}^{+}(\beta, h) .
$$

The analogous quantity defined using "minus" boundary conditions is denoted by $F_{L, M}^{-}(\beta, h)$.

\section{The New Results}

All our new results depend on the following theorem, which is proved in the next section: 


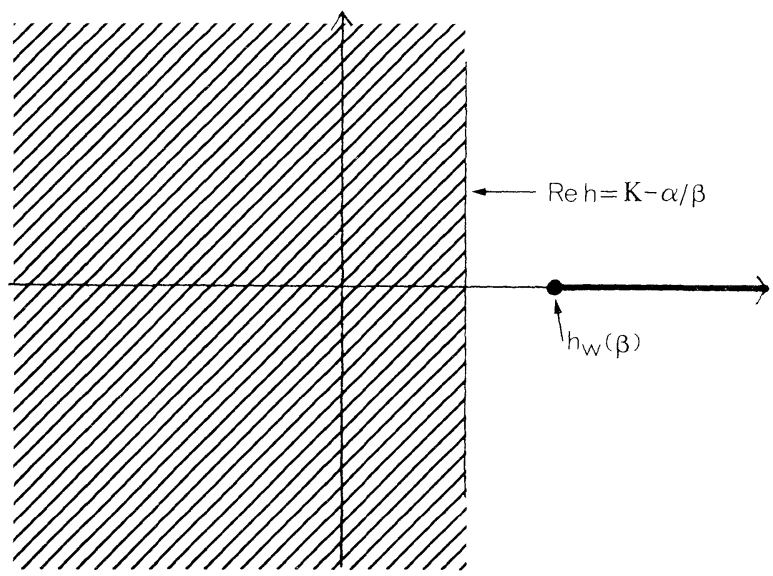

Fig. 1. Part of the complex $h$ plane. The domain of analyticity of $F^{-}(\beta, h)$ is known to include the shaded region and the heavy part of the real $h$-axis

Theorem. The surface free energies $F^{+}(\beta, h)$ and $F^{-}(\beta, h)$ are analytic functions of $h$ in the region of the complex $h$ plane defined by

$$
|\operatorname{Re} h|<K-\alpha / \beta \text {, }
$$

where $\alpha=\ln [3(1+2 e)]=2.96 \ldots$

The proof of this theorem is given in Sect. 4 .

Using the theorem we can investigate the domains of analyticity of $F^{+}$and $F^{-}$ in the complex $h$ plane. First of all, as a consequence of the Lee-Yang theorem [6], $F^{+}$is analytic in $h$ for $\operatorname{Re} h>0$. Combining this fact with the above theorem we see that $F^{+}(\beta, h)$ is analytic in the union of the right half-plane $h>0$ and the strip in which (3.1) holds; that is to say, in the half-plane $\operatorname{Re} h>-(K-\alpha / \beta)$. By symmetry, since

$$
F^{-}(\beta, h)=F^{+}(\beta,-h),
$$

$F^{-}(\beta, h)$ is analytic for $\operatorname{Re} h<K-\alpha / \beta$.

Secondly, it can be shown [2] that, for $h \geqq 0$,

$$
0 \leqq F^{-}(\beta, h)-F^{+}(\beta, h) \leqq \tau(\beta),
$$

where $\tau(\beta)$ is the free energy of the interface between bulk "plus" and "minus" phases when there is no wall. Let $h_{w}(\beta)$ be the value of the magnetic field at which wetting takes place; it is shown in [2] that this can be characterized by

$$
h_{w}(\beta)=\inf \left\{h \geqq 0: F^{-}(\beta, h)-F^{+}(\beta, h)=\tau(\beta)\right\} .
$$

Thus for real values of $h$ exceeding $h_{w}(\beta)$ we have $F^{-}(\beta, h)=F^{+}(\beta, h)+\tau(\beta)$, so that $F^{-}$is real analytic in $h$ because $F^{+}$is. Likewise $F^{+}$is real analytic in $h$ for $h<-h_{w}(\beta)$.

The domain of analyticity for $F^{-}$implied by these results is shown in Fig. 1.

One way we can use our theorem is to show that

$$
K-\alpha / \beta \leqq h_{w}(\beta) \text {. }
$$


For, if the reverse were true we could arrive at a contradiction. On the one hand, by (3.4), $F^{-}-F^{+}$would have the constant value $\tau(\beta)$ along the line segment $h_{w}(\beta)<h<K-\alpha / \beta$, which lies in the joint domain of analyticity of $F^{+}$and $F^{-}$; and it would then follow by analytic continuation that $F^{+}(\beta, h)-F^{-}(\beta, h)$ has the value $\tau(\beta)$ throughout this joint domain of analyticity, and in particular at $h=0$. Since (3.2) implies $F^{+}(\beta, 0)-F^{-}(\beta, 0)=0$, we would have $\tau(\beta)=0$. But, on the other hand, the falsity of (3.5) would also imply $K-\alpha / \beta>0$, and therefore $\beta K>\alpha=2.96 \ldots$. Since $\beta_{c} K=0.44 \ldots$ in two dimensions and is even smaller in more dimensions, it would follow that $\beta>\beta_{c}$, and hence [7] that $\tau(\beta)>0$. Thus if (3.5) were false we would have both $\tau(\beta)=0$ and $\tau(\beta)>0$; so (3.5) must be true.

For three or more dimensions, it is known [2] that for large enough $\beta$ there is a magnetic field $h^{*}(\beta)$ at which the quantity

$$
(d / d h)\left[F^{+}(\beta, h)-F^{-}(\beta, h)\right]
$$

(which can be interpreted as an order parameter) is discontinuous - a first-order phase transition. Our theorem thus shows that $K-\alpha / \beta$ is also a lower bound on $h^{*}(\beta)$.

\section{Proof of the Theorem}

The theorem is proved by a low-temperature cluster expansion in terms of contours. To obtain this expansion we first replace $H_{A}$ by the equivalent Hamiltonian

$$
\tilde{H}_{A}^{+}=-K \sum_{\langle i j\rangle}(s(i) s(j)-1)-h \sum_{i \in \Sigma}(s(i)-1),
$$

where $s(i)=1$ if $i$ is not in $\Lambda$, so that only the terms for which $i$ or $j$ is in $\Lambda$ contribute. We also define the corresponding equivalent Hamiltonians for the Ising models in $\Lambda^{\prime}$ and $\Omega$. Let $\widetilde{Z}_{L, M}^{+}$and $\widetilde{Q}_{L, M}^{+}$be the corresponding new partition functions on $\Lambda$ (or $\left.\Lambda^{\prime}\right)$ and on $\Omega$. In consequence of (2.4) we have

$$
F_{L, M}^{+}(\beta, h)=\frac{-1}{2 \beta(2 L+1)^{d-1}} \ln \frac{\left(\tilde{Z}_{L, M}^{+}\right)^{2}}{\widetilde{Q}_{L, M}^{+}}+\frac{1}{2} K-h .
$$

Next we show how to represent configurations on the finite lattices $\Lambda, \Lambda^{\prime}$, and $\Omega$ by means of contours. For simplicity we only consider the case $d=2$, though the generalization to $d \geqq 3$ is straightforward. Let $\{s(i)\}$ be any configuration. For each nearest-neighbour pair $\langle i j\rangle$ with $s(i) s(j)=-1$, we draw a unit line segment perpendicularly bisecting the bond $\langle i j\rangle$ (see Fig. 2); and for each site $i$ in the layer $\Sigma$ for which $s(i)=-1$ we draw a unit line segment in the line $z=\frac{1}{2}$ as close as possible to the site $i$. The totality of all these line segments is a subset of the dual lattice $A^{*}$ whose vertices have half-integral co-ordinates. The contours are defined as the connected components of this subset, and are denoted by $\gamma_{1} \ldots \gamma_{n}$. A configuration is uniquely determined by its set of contours.

For a given contour $\gamma$, let $|\gamma|$ denote its length, i.e. the total number of segments comprising it, and let $b(\gamma)$ be the number of segments (if any) lying in the boundary line $z=\frac{1}{2}$. According to (4.1), each segment of $\gamma$ in the line $z=\frac{1}{2}$ contributes an 


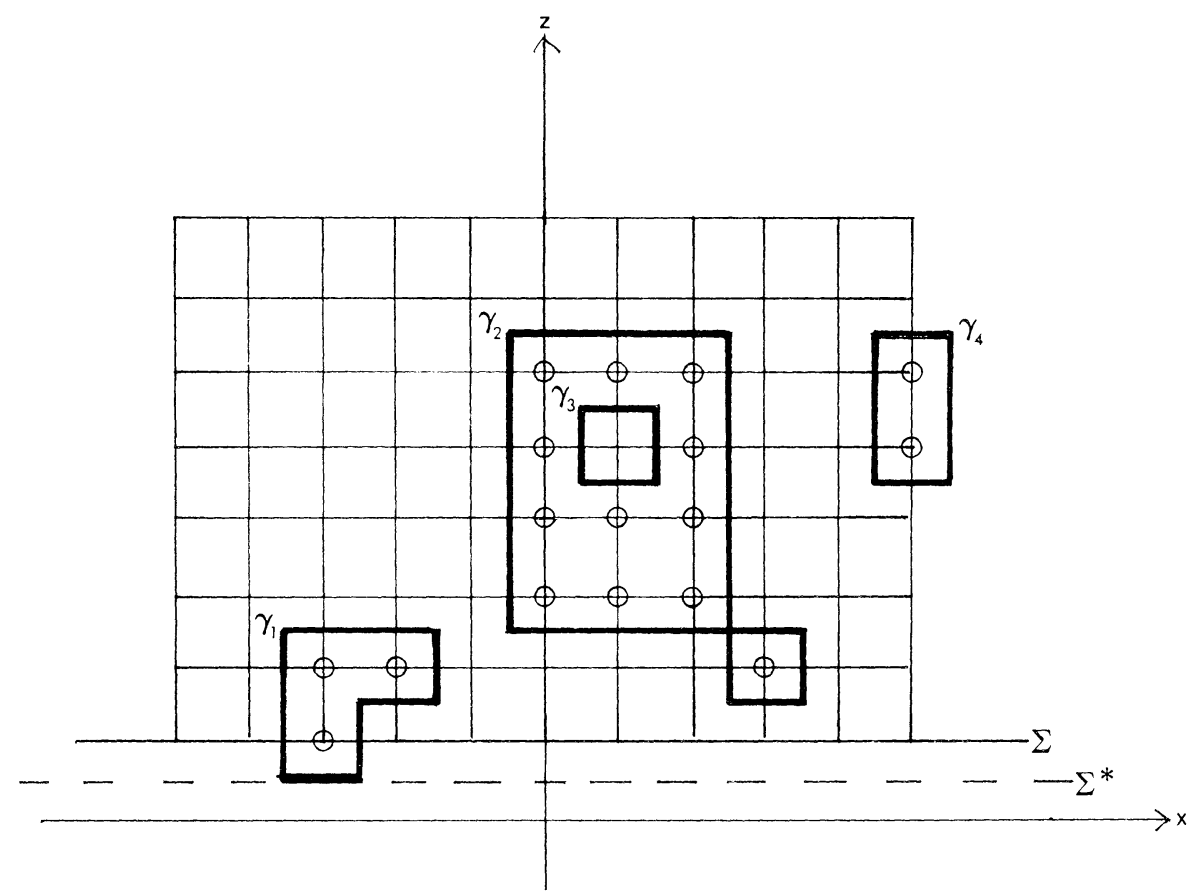

Fig. 2. A configuration of $A(5,8)$ and the corresponding contours. The circles mark the sites occupied by "minus" spins; sites with "plus" spins are unmarked. The co-ordinate axes and the layers $\Sigma$ (in which $z=1$ ) and $\Sigma^{*}$ (in which $z=\frac{1}{2}$ ) are also shown

amount $2 h$ to the energy $\tilde{H}_{A}^{+}$, and each segment not in this line contributes an amount $2 K$; so the total contribution of the contour $\gamma$ to the energy is

$$
E(\gamma)=2 h b(\gamma)+2 K(|\gamma|-b(\gamma))
$$

Since the parts of $\gamma$ lying in the line $z=\frac{1}{2}$, if any, are straight, we have $|\gamma|-b(\gamma)>b(\gamma)$, so that

$$
2(|\gamma|-b(\gamma))>|\gamma|>2 b(\gamma)
$$

It follows that the Boltzmann factor

$$
\zeta(\gamma):=\exp (-\beta E(\gamma))
$$

satisfies, for any complex $h$,

$$
|\zeta(\gamma)| \leqq \exp (-\beta(K-|\operatorname{Re} h|)|\gamma|) .
$$

A similar definition of contours can be applied to the reflected finite lattice $\Lambda^{\prime}$; in this case all the contours lie in the half-plane $z \leqq \frac{1}{2}$ instead of $z \geqq \frac{1}{2}$, but the formulas (4.3)-(4.6) still apply. The same thing can also be done for the lattice $\Omega$; in that case contours crossing the line $z=\frac{1}{2}$ also appear, and the Boltzmann factor is simply

$$
\zeta^{\prime}(\gamma):=\exp (-2 \beta K|\gamma|)
$$


At low temperatures, the likely configurations have rather few contours, and an expansion in powers of the Boltzmann factors $\zeta(\gamma)$ can be used. The expansion is

$$
\tilde{Z}_{L, M}^{+}=1+\sum_{n \geqq 1} \frac{1}{n !} \sum_{\gamma_{1} \subset \Lambda^{*}} \ldots \sum_{\gamma_{n} \subset \Lambda^{*}} \phi\left(\gamma_{1}, \ldots, \gamma_{n}\right) \prod_{i=1}^{n} \zeta\left(\gamma_{i}\right),
$$

where the notation $\gamma \subset A^{*}$ means that $\gamma$ is a contour on the dual lattice of $\Lambda$, and $\Phi\left(\gamma_{1}, \ldots, \gamma_{n}\right)$ is defined to equal 1 whenever the contours in the family $\left(\gamma_{1}, \ldots, \gamma_{2}\right)$ are all disjoint and to equal 0 otherwise (e.g. if $\gamma_{1}=\gamma_{2}$ ).

A formal rearrangement of the series (4.8) gives [8]

$$
\tilde{Z}_{L, M}^{+}=\exp \left(\sum_{n \geqq 1} \frac{1}{n !} \sum_{\gamma_{1} \subset \Lambda^{*}} \ldots \sum_{\gamma_{n} \subset A^{*}} \Phi^{T}\left(\gamma_{1}, \ldots, \gamma_{n}\right) \prod_{i=1}^{n} \zeta\left(\gamma_{i}\right)\right),
$$

with $\Phi^{T}$ defined by

$$
\Phi^{T}\left(\gamma_{1}, \ldots, \gamma_{n}\right)=\left\{\begin{array}{lll}
1 & \text { if } & n=1 \\
\sum_{g \in C_{n}} \prod_{i j\rangle \in g} f\left(\gamma_{i}, \gamma_{j}\right) & \text { if } & n \geqq 2
\end{array},\right.
$$

where $C_{n}$ is the set of all connected graphs on $\{1, \ldots, n\}$ and $f\left(\gamma_{i}, \gamma_{j}\right)$ is defined to be -1 if the contours $\gamma_{i}$ and $\gamma_{j}$ meet and to be 0 if they do not. In particular $\Phi^{T}(\ldots)$ is zero if the set $U\left(\gamma_{1}, \ldots, \gamma_{n}\right)$ is not connected.

Theorem 2 of Cammarota [8] shows that the formal rearrangement giving (4.9) is justified, and that the rearranged series is absolutely convergent, provided that for every bond (unit line segment) $x$ in $A^{*}$ the inequality

$$
\sum_{\substack{\gamma \ni x \\|\gamma|=n}}|\zeta(\gamma)| \leqq \varepsilon^{n}
$$

holds for all integers $n \geqq 1$, with

$$
\varepsilon<1 /(1+2 e) .
$$

Since the number of contours of length $n$ containing a given segment $x$ is at most $3^{n}$, the estimate (4.6) implies that (4.11) holds, with

$$
\varepsilon=3 \exp (-\beta(K-|\operatorname{Re} h|)) \text {. }
$$

Condition (3.1) of our theorem then ensures that (4.12) holds as well.

In just the same way, noting that $\left|\zeta^{\prime}(\gamma)\right|<|\zeta(\gamma)|$ by (4.6) and (4.7), we can show that the corresponding series for $\widetilde{Q}_{L, M}^{+}$,

$$
\widetilde{Q}_{L, M}^{+}=\exp \left(\sum_{n \geqq 1} \frac{1}{n !} \sum_{\gamma_{1} \subset \Omega^{*}} \ldots \sum_{\gamma_{n} \subset \Omega^{*}} \Phi^{T}\left(\gamma_{1}, \ldots, \gamma_{n}\right) \prod_{i=1}^{n} \zeta^{\prime}\left(\gamma_{i}\right)\right)
$$

is valid under the conditions of our theorem.

Now we can evaluate the surface free energy, using (4.2), (4.9), (4.14), and the mirror image of (4.9) in terms of contours in $\Lambda^{\prime}$. The resulting formula (taking $d=2$ ) is

$$
\begin{aligned}
F_{L, M}^{+}(\beta, h)= & \frac{1}{2} K-h-\frac{1}{2 \beta(2 L+1)} \sum_{n \geqq 1} \frac{1}{n !} \sum_{\gamma_{1} \subset \Omega^{*}} \ldots \sum_{\gamma_{n} \subset \Omega^{*}} \Phi^{T}\left(\gamma_{1}, \ldots, \gamma_{n}\right) \\
& \times\left[\prod_{i=1}^{n} \zeta\left(\gamma_{i}\right) \chi_{\Lambda}\left(\gamma_{i}\right)+\prod_{i=1}^{n} \zeta\left(\gamma_{i}\right) \chi_{\Lambda^{\prime}}\left(\gamma_{i}\right)-\prod_{i=1}^{n} \zeta^{\prime}\left(\gamma_{i}\right)\right],
\end{aligned}
$$


where $\chi_{\Lambda}(\gamma)$ is defined to be 1 if $\gamma$ is in $\Lambda^{*}$ and to be 0 otherwise, while $\chi_{\Lambda^{\prime}}$ is the corresponding function for $\Lambda^{*}$.

As we have noted earlier, in order for $\Phi^{T}\left(\gamma_{1}, \ldots, \gamma_{n}\right)$ to be non-zero, it is necessary for the set $\Gamma:=\bigcup_{i}\left(\gamma_{i}\right)$ to be connected. Moreover, by (4.3), (4.5), and (4.7), the expression in square brackets is zero if this connected set lies entirely in one of the half-spaces $z>\frac{1}{2}$ or $z<\frac{1}{2}$. Thus the only non-vanishing terms of the sum are those for which $\Gamma$ is connected and meets the line $z=\frac{1}{2}$. Noting again that $\left|\zeta^{\prime}(\gamma)\right|$ $<|\zeta(\gamma)|$, we see that the series in (4.15) is dominated by three times the sum

$$
\sum_{n \geqq 1} \frac{1}{n !} \sum_{\gamma \cap \Sigma^{*} \neq \emptyset} \sum_{\substack{\gamma_{1}, \ldots, \gamma_{n} \subset \Omega^{*} \\ \exists \gamma_{1}=\gamma}}\left|\Phi^{T}\left(\gamma_{1}, \ldots, \gamma_{n}\right)\right|\left|\zeta\left(\gamma_{1}\right)\right| \ldots\left|\zeta\left(\gamma_{n}\right)\right|,
$$

where $\Sigma^{*}$ denotes the line $z=\frac{1}{2}$. Using Eq. (1.20) of [8] and the estimate (4.11) for $\zeta$, we see that under the conditions of our theorem the sum in (4.16) is bounded by

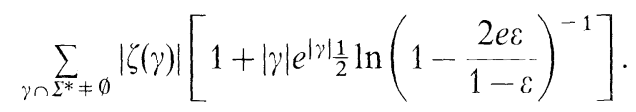

This in turn is bounded by

$$
\sum_{x} \sum_{\gamma \ni x} \text { (same summand), }
$$

where the summation over $x$ goes over all bonds $x$ in $\Omega^{*}$ which meet $\Sigma^{*}$. Equations (4.11) and (4.12) now ensures that the summation over $\gamma$ in (4.18) is dominated by the convergent series

$$
C:=\sum_{n} \varepsilon^{n}\left[1+n e^{n} \frac{1}{2} \ln \left(1-\frac{2 e \varepsilon}{1-\varepsilon}\right)^{-1}\right] .
$$

Hence the series in (4.16) is dominated by $C$ times the number of bonds $x$ in $\Omega^{*}$ which meets $\Sigma^{*}$, and therefore by $3 C(2 L+1)$. It follows that the terms in that series, after division by $(2 L+1)$, are bounded by the terms in a convergent series whose terms are independent of $L$ and $M$; hence, by the Weierstrass $M$-test applied to the series (4.15), as $L$ and $M$ become large the functions $F_{L, M}^{+}(\beta, h)$ tend uniformly to the function obtained by taking the term-by-term limit, which is

$$
\begin{aligned}
F^{+}(\beta, h)= & \frac{1}{2} K-h-\frac{1}{2 \beta} \sum_{n \geqq 1} \frac{1}{n !} \sum_{\gamma_{1}, \ldots, \gamma_{n}} \Phi^{T}\left(\gamma_{1}, \ldots, \gamma_{n}\right) \\
& \times\left[\prod_{i=1}^{n} \zeta\left(\gamma_{i}\right) \chi_{\mathbb{L}}\left(\gamma_{i}\right)+\prod_{i=1}^{n} \zeta\left(\gamma_{i}\right) \chi_{\mathbb{L}} \cdot\left(\gamma_{i}\right)-\prod_{i=1}^{n} \zeta^{\prime}\left(\gamma_{i}\right)\right],
\end{aligned}
$$

where $\chi_{\mathbb{L}}(\gamma)$ is 1 if $\gamma$ is in the dual lattice of $\mathbb{L}$ and is 0 otherwise, and $\chi_{\mathbb{L}}$, is defined similarly. The inner summation is over all equivalence classes of families of $n$ contours in $\left(\mathbb{Z}^{d}\right)^{*}$, the equivalence being under translations within $\Sigma$, that is ones perpendicular to the $z$ direction. Since the terms in the series are all analytic in $h$ and the convergence of the series is uniform in the region of the complex $h$ plane defined by (3.1), the sum of the series, which is $F^{+}(\beta, h)$, is analytic in that region.

The corresponding result for $F^{-}$now follows immediately from the symmetry relation (3.2), and so the proof of the theorem is complete. 


\section{Discussion}

Although the proof given above refers to the two-dimensional case, it can be generalized without difficulty to three or more dimensions.

The proof is also extended without difficulty to the case where the interaction between neighbouring spins in the surface (i.e. in $\Sigma$ ) is different from that in the other bonds. If the interaction in the surface is $J$, while that in the other bonds is $K$ as before, then for $J>K$ the theorem is still true as stated. For the case $0<J<K$ the theorem holds provided the inequality (3.1) is replaced by

$$
|\operatorname{Re} h| \leqq J-\alpha / \beta \text {. }
$$

In the case where $J=0$, this last result provides no information, but one can then sum over all the spins in $\Sigma$ and reduce the analysis to the case where $J=K$ and $h$ is replaced by $h_{\text {ef }}$, where

$$
\tanh \left(h_{\mathrm{ef}}\right)=\tanh (K) \tanh (h),
$$

as in p. 66 of [2]. Thus, for the case $J=0$, the theorem holds with (3.1) replaced by

$$
\left|\operatorname{Re} h_{\mathrm{ef}}\right| \leqq K-\alpha / \beta \text {. }
$$

Acknowledgements. This work was made possible by financial support from the Science and Engineering Research Council. C. Pfister thanks the Department of Mathematics at Heriot-Watt University for its kind hospitality.

\section{References}

1. Abraham, D.B.: Soluble model with a roughening transition for a planar Ising ferromagnet. Phys. Rev. Lett. 44, 1165-1168 (1980)

2. Fröhlich, J., Pfister, C.-E.: Semi-infinite Ising model. II. The wetting and layering transitions. Commun. Math. Phys. 112, 51-74 (1987)

3. Fröhlich, J., Pfister, C.-E.: The wetting and layering transitions in the half-infinite Ising model. Europhys. Lett. 3, 845-852 (1987)

4. McCoy, B.M., Wu, T.-T.: The two-dimensional Ising model. Cambridge, MA: Harvard University Press 1973

5. Fröhlich, J., Pfister, C.-E.: Semi-infinite Ising model. I. Thermodynamic functions and phase diagram in absence of magnetic field. Commun. Math. Phys. 109, 493-523 (1987)

6. Lee, T.D., Yang, C.N.: Statistical theory of equations of state and phase transitions. II. Lattice gas and Ising model. Phys. Rev. 87, 410-418 (1952)

7. Lebowitz, J.L., Pfister, C.-E.: Surface tension and phase coexistence. Phys. Rev. Lett. 46, 1031-1033 (1981)

8. Cammarota, C.: Decay of correlations for infinite range interactions in unbounded spin systems. Commun. Math. Phys. 85, 517-528 (1982)

Communicated by J. Fröhlich

Received November 26, 1987 
